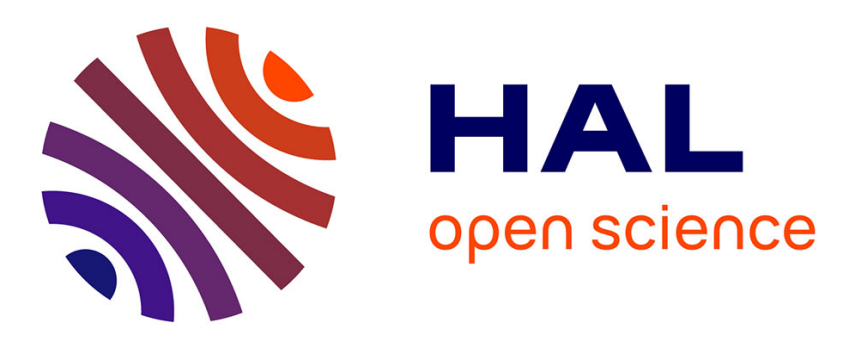

\title{
Operational Modal Analysis and Wavelet Transformation for Damage Identification in Wind Turbine Blades
}

Martin Dalgaard Ulriksen, Dmitri Tcherniak, Poul Henning Kirkegaard, Lars

Damkilde

\section{To cite this version:}

Martin Dalgaard Ulriksen, Dmitri Tcherniak, Poul Henning Kirkegaard, Lars Damkilde. Operational Modal Analysis and Wavelet Transformation for Damage Identification in Wind Turbine Blades. EWSHM - 7th European Workshop on Structural Health Monitoring, IFFSTTAR, Inria, Université de Nantes, Jul 2014, Nantes, France. hal-01021191

\section{HAL Id: hal-01021191 \\ https://inria.hal.science/hal-01021191}

Submitted on 9 Jul 2014

HAL is a multi-disciplinary open access archive for the deposit and dissemination of scientific research documents, whether they are published or not. The documents may come from teaching and research institutions in France or abroad, or from public or private research centers.
L'archive ouverte pluridisciplinaire HAL, est destinée au dépôt et à la diffusion de documents scientifiques de niveau recherche, publiés ou non, émanant des établissements d'enseignement et de recherche français ou étrangers, des laboratoires publics ou privés. 


\title{
Operational Modal Analysis and WaVelet Transformation FOR DAMAGE IDENTIFICATION IN WIND TURBINE BLADES
}

\author{
Martin D. Ulriksen ${ }^{1}$, Dmitri Tcherniak ${ }^{2}$, Poul H. Kirkegaard ${ }^{1}$, Lars Damkilde ${ }^{1}$ \\ ${ }^{1}$ Dept. of Civil Engineering, Aalborg University, Sohngaardsholmsvej 57, Aalborg, Denmark \\ ${ }^{2}$ Brüel \& Kjarr Sound \& Vibration Measurement A/S, Skodsborgvej 307, Narum, Denmark \\ mdu@ civil.aau.dk
}

\begin{abstract}
The presented study demonstrates an application of a previously proposed modal and wavelet analysis-based damage identification method to a wind turbine blade. A trailing edge debonding was introduced to a SSP $34 \mathrm{~m}$ blade mounted on a test rig. Operational modal analysis (OMA) was conducted to obtain mode shapes for undamaged and damaged states of the blade. Subsequently, the mode shapes were analyzed with one-dimensional continuous wavelet transformations (CWTs) for damage identification. The basic idea of the method is that structural damage will introduce local mode shape irregularities which are captured in the CWT by significantly magnified transform coefficients, thus providing combined damage detection, localization, and size assessment. It was found that due to the nature of the proposed method, the value of the identification results highly depends on the number of employed measurement points. Since only a limited number of measurement points were utilized in the experiments, valid damage identification can only be obtained when employing high-frequency modes.
\end{abstract}

KEYWORDS : Wind Turbine Blades, Debonding, Wavelet Transformation, Operational Modal Analysis.

\section{INTRODUCTION}

While failure can happen in any structural component of the wind turbine, one of the most common and critical components to fail is the blade [1]. Here, several damage types can occur [2], and in extreme situations, the entire blade can be destroyed. Since the blades account for 15-20\% of the total wind turbine costs [3], a great need in a dedicated blade structural health monitoring (SHM) system, facilitating 3-level damage identification, i.e. detection, localization, and assessment, c.f. [4], was identified. The different proposed blade SHM methods utilize a wide range of quantities like temperature, noise, and vibration. As described in [5], the applicability of each of these methods is primarily tested on the basis of controlled laboratory tests, or by finite element (FE) simulations. With both of these methods, significant simplifications and idealizations are made with regard to, i.a., environmental effects. Under these conditions, several simple methods comparing pre- and post-damage quantity values, e.g. modal parameters, have exhibited potential for damage detection. However, under conditions that are more realistic, these methods are inapplicable since environmental effects and noise contamination typically will conceal the direct changes of the previously mentioned quantities. As an example, it is stated in [6] how environmental effects can account for up to $5 \%$ changes in eigenfrequencies, which, as is also demonstrated in the present study, cannot be expected to be exceeded by damage-induced eigenfrequency changes. Due to the general inadequacy of the simple methods, current research within the field of blade SHM is leveled mainly at developing more sophisticated and robust methods that, for example, include advanced signal processing or statistical analysis, see e.g. [7].

The present paper documents the application of the early-proposed vibration-based method, employing continuous wavelet transformation (CWT) of spatial mode shape signals for damage detec- 
tion, localization, and assessment on a full-scale wind turbine blade. In [8,9], similar studies were conducted as CWTs were used to identify different cracks in residential-sized wind turbine blades. In these studies, the mode shapes were derived through experimental modal analysis (EMA), where an impact hammer was used for excitation. In the present study, operational modal analysis (OMA) is used to obtain the mode shapes and this means we can examine the applicability of the proposed damage identification method for blades under conditions that resemble in-service conditions more closely. Additionally, this paper uses a simple damage indicator, inspired by modal assurance criterion (MAC), which expresses the similarity between pre- and post-damage wavelet transforms.

The paper is organized as follows: the first section describes the test object, the damage, the measurement system, and the conducted experiments; while the following section gives the basics of CWT and describe the application of the method to the mode shapes.

\section{OMA OF SSP 34 M BLADE}

\subsection{Test object}

A $34 \mathrm{~m}$ long wind turbine blade, designed and manufactured by SSP, was mounted on an indoor test rig as shown in Figure 1. The SSP $34 \mathrm{~m}$ blade is made from epoxy fiberglass and has a mass of $4,600 \mathrm{~kg}[10]$.

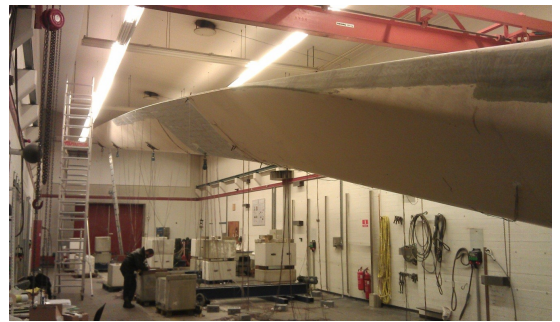

(a)

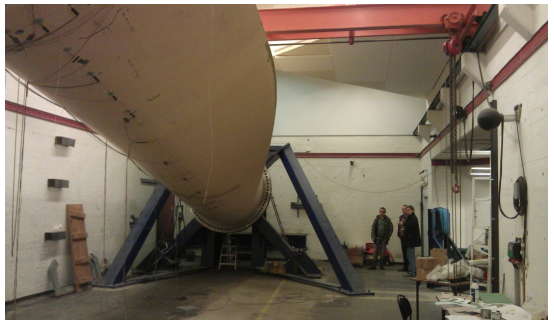

(b)

Figure 1 : SSP 34 m blade mounted on the test rig. (a) Root-to-tip view. (b) Tip-to-root view.

\subsection{Measurement system}

The measurement system consisted of 20 triaxial accelerometers (Brüel \& Kjær Type 4524-B) mounted on leading and trailing edges at ten stations along the blade, see Figure 2(a). The distances between the neighboring stations differed along the blade, from $4,100 \mathrm{~mm}$ at the blade root to $2,000 \mathrm{~mm}$ at the tip.

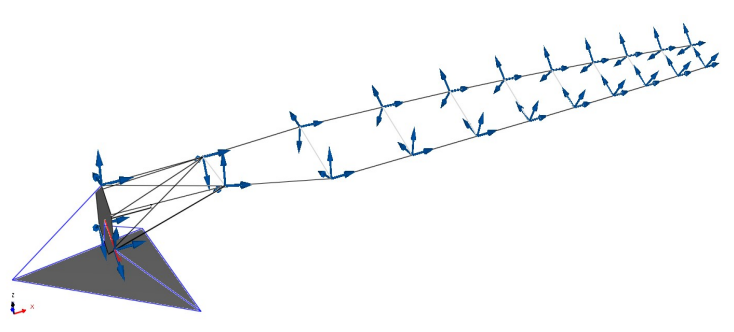

(a)

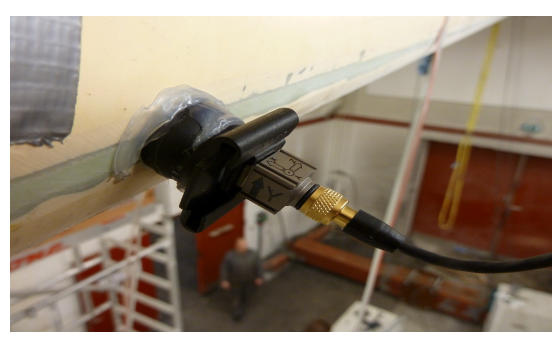

(b)

Figure 2: Accelerometer mounting on SSP 34 m blade. (a) Accelerometer location and orientation. (b) Utilized accelerometers (Brüel \& Kjær Type 4524-B) mounted on a swivel base (Brüel \& Kjær UA-1473). 
Location and orientation of the accelerometers have a key importance for getting correct mode shapes: their location and orientation in reality should be as close as possible to the coordinates and orientation of measurement DOFs documented in the modal analysis software. In this project, this was achieved by using a dedicated template that was aligned against the foil profile at the stations, and the accelerometers were fixed on swivel bases according to the template. In Figure 2(b), a picture of an accelerometer mounted on a swivel base is shown.

Due to large distances between measurement points, it was advantageous using a distributed data acquisition system based on five, 12-channel, Brüel \& Kjær LAN-XI modules Type 3053-B-120. Each module served two stations, i.e. four triaxial accelerometers, and was placed near the sensors, therefore keeping the length of the accelerometer cables minimal. The modules were powered via an Ethernet cable, which was also used for data transfer and modules synchronization.

\subsection{Data acquisition}

According to OMA assumptions, the excitation is required to be distributed over the entire structure, it needs to be uncorrelated, and have a flat spectra in the frequency range of interest. The blade was excited by several people hitting the blade at a number of positions with heavy wooden sticks wrapped in foam.

Data from the accelerometers was recorded during approximately seven minutes, corresponding to at least 500 oscillations at the lowest frequency of interest, which was identified as approximately $1.3 \mathrm{~Hz}$.

\subsection{Damage}

To ensure that the damage introduced to the blade model is realistic, a study of typical structural blade damages was conducted and some of the critical ones were chosen for further work.

In [2], 15 blades of $300 \mathrm{~kW}$ wind turbines and 81 blades of $100 \mathrm{~kW}$ wind turbines, all with a lifetime range of between 16 and 19 years, were inspected. As a result of these inspections, several different damage types were found and subsequently categorized into the three overall groups; cracks, edge damages, and debondings. Of these damage types, the most frequently occurring and severe were debondings plus longitudinal and transverse cracks near and at the blade edges. These all required structural repairs for the blades to operate properly, c.f. [2]. Consequently, in the present study we decided to treat one of these specific damage types, namely a trailing edge debonding, see Figure 3.

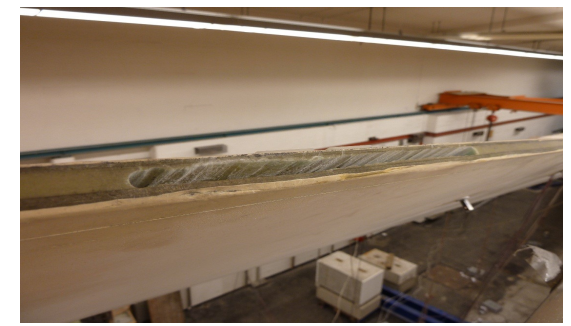

(a)

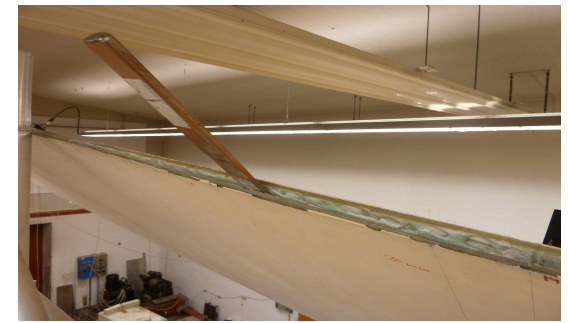

(b)

Figure 3 : Debonding introduced in the trailing edge of an SSP $34 \mathrm{~m}$ blade. (a) Original drilled debonding. (b) Extended debonding.

The debonding was first introduced by drilling a series of holes perpendicular to the trailing edge, such that the glue connecting the shells was removed to yield the damage depicted in Figure 3(a). Subsequently, the debonding was gradually extended using a hammer and a chisel, resulting in the 1,200 $\mathrm{mm}$ long debonding shown in Figure 3(b). The extended debonding was located between $18,800 \mathrm{~mm}$ and $20,000 \mathrm{~mm}$ from the blade root. 


\subsection{Experiment and analysis}

First, a series of OMA tests was performed on the undamaged blade and then the blade with different lengths of debonding. The damage was repaired according to the blade guidelines, and one more OMA test was conducted.

Since some additional, no-visible, inner-damage could occur when operating with the heavy hammer and chisel, it was decided to focus on two states; the state just before the damage was repaired, and the state right after the repair. For each of these states, a 20 minute long data set was available. The data set was split into three equal segments, each about seven minutes long, and OMA was performed on each of the segments. OMA was also performed on the entire 20 minute data sets to see if more data would enhance the OMA algorithm performance. Therefore, in total, eight states were considered, with states 1-4 and 5-8 characterizing the 'damaged' state and 'undamaged' state, respectively.

Brüel \& Kjær OMA software Type 7760 was employed, and the stochastic subspace identification (SSI) algorithm was applied to the data. The ten lowest modes were identified for all eight states. In Table 1, the mode names and their frequencies (the mean and $95 \%$ confidence estimated from four averages) are presented. Additionally, the ten mode shapes are plotted in Figure 4 for all eight states.

Table 1 : First ten modes for damaged and undamaged states of SSP $34 \mathrm{~m}$ blade.

\begin{tabular}{clccccc}
\hline \multirow{2}{*}{ Mode number } & \multirow{2}{*}{ Mode type } & \multicolumn{3}{c}{ Frequencies [Hz] } \\
\cline { 3 - 4 } & & \multicolumn{2}{c}{ Damaged } & & \multicolumn{2}{c}{ Undamaged } \\
\cline { 3 - 4 } \cline { 7 - 8 } & & Mean & Confid. & & Mean & Confid. \\
\hline 1 & 1st flap & 1.35 & 0.01 & & 1.36 & 0.01 \\
2 & 1st edge & 1.86 & 0.01 & & 1.86 & 0.01 \\
3 & 2nd flap & 4.21 & 0.01 & & 4.21 & 0.00 \\
4 & 2nd edge & 7.12 & 0.01 & & 7.12 & 0.00 \\
5 & 3rd flap & 9.17 & 0.01 & & 9.19 & 0.06 \\
6 & 1st torsion & 12.37 & 0.01 & & 12.40 & 0.02 \\
7 & 3rd edge + 4th flap & 14.19 & 0.06 & & 14.16 & 0.10 \\
8 & 4th flap + 3rd edge & 14.98 & 0.01 & & 14.99 & 0.01 \\
9 & Complex shape & 15.33 & 0.02 & & 15.48 & 0.03 \\
10 & Complex shape & 17.99 & 0.05 & & 18.08 & 0.04 \\
\hline
\end{tabular}

\section{DAMAGE IDENTIFICATION IN SSP 34 M BLADE}

Using the OMA method presented in Section 1., the modal parameters for the SSP $34 \mathrm{~m}$ blade were extracted. Even though the experiment was conducted in a well controlled test-rig environment, the changes in the natural frequencies due to the damage are comparable with the dispersion due to measurement noise, see Table 1. Further, direct visual comparison of the mode shapes plotted in Figure 4 could not provide valid information to facilitate damage identification. Consequently, a more sophisticated and robust method, in the form of CWT, was employed.

\subsection{Basics of CWT}

The idea of the proposed damage identification method is to apply CWT to the spatial mode shape signals and thereby identify damage-induced irregularities in these signals. In this section, focus 

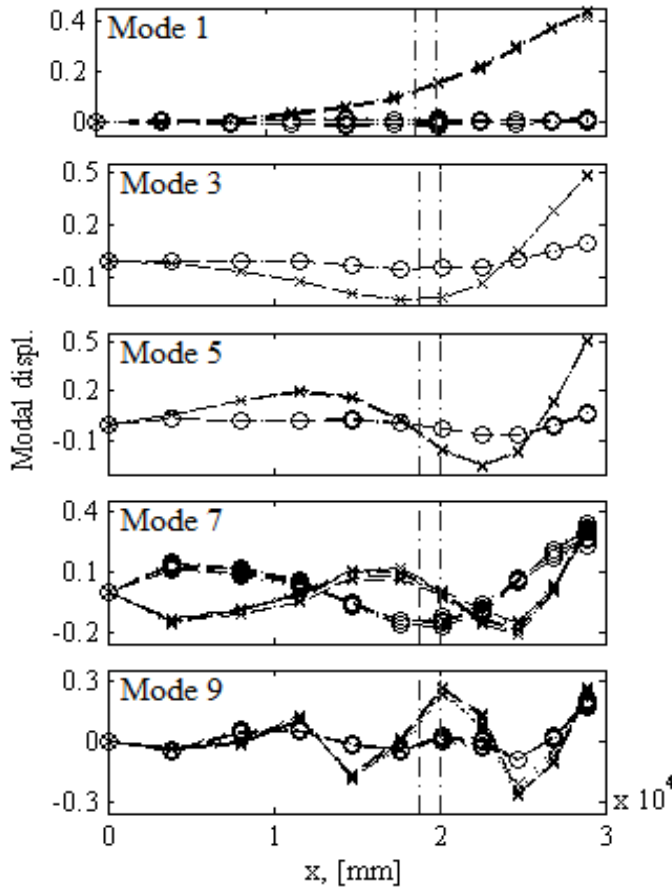
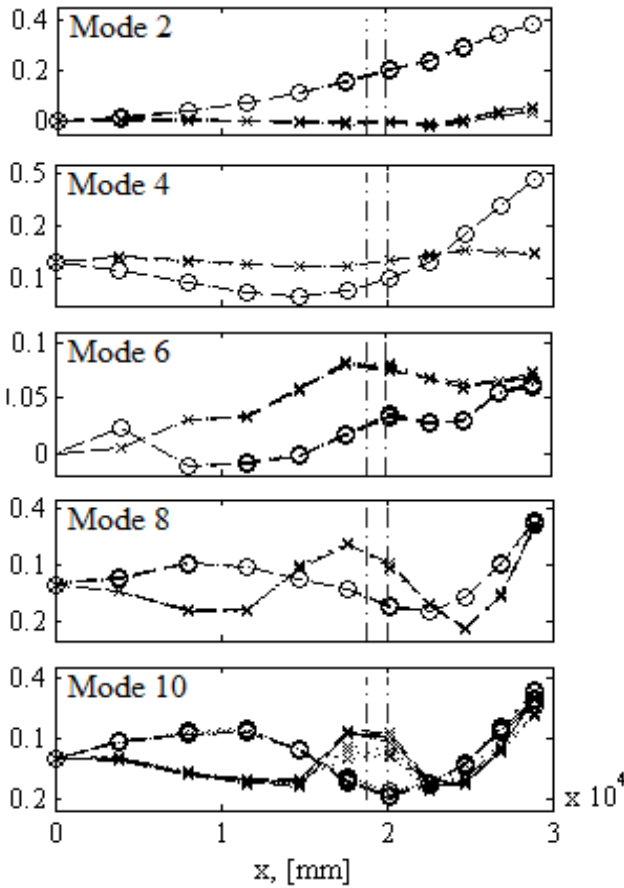

Figure 4 : Flapwise $(\times)$ and edgewise (o) components of the first ten mode shapes of damaged $(\cdots)$ and undamaged (--) SSP $34 \mathrm{~m}$ blade. The dashed vertical lines mark the debonding location.

will be on describing the basics of CWT and its applicability in damage identification. The latter includes employment of mode shape signal interpolation and extrapolation procedures for improving the wavelet transform as a damage indicator.

By comparing the spatial mode shape signal, $f(x) \in \mathbf{L}^{2}(\mathbb{R})$, to the wavelet function, $\psi(x) \in$ $\mathbf{L}^{2}(\mathbb{R})$, at various wavelet scales, $a \in \mathbb{R}^{+}$, and positions, $b \in \mathbb{R}$, the CWT

$$
W f(a, b)=\frac{1}{\sqrt{a}} \int_{\mathbb{R}} f(x) \psi^{*}\left(\frac{x-b}{a}\right) \mathrm{d} x
$$

is obtained as the inner product of $f(x)$ and the complex conjugated, indicated with the superscript *, of the so-called wavelet family. The wavelet family consists of functions constructed from dilations and translations of $\psi$, that is

$$
\psi_{a, b}(x)=\frac{1}{\sqrt{a}} \psi\left(\frac{x-b}{a}\right)
$$

thus large values of $a$ correspond to big wavelets and consequently coarse features of $f(x)$, while low values of $a$ correspond to fine details of $f(x)$. In Equation (2), the factor $\frac{1}{\sqrt{a}}$ is applied as a normalization operator such $\left\|\psi_{a, b}(x)\right\|=\|\psi(x)\|$.

\subsection{CWT for damage identification}

Because of the effectively limited duration of the waveform, signals with abrupt changes can be better analyzed with a wavelet than with a smooth sinusoid. This is exploited in the proposed damage identification method, where the idea is that structural damage in the blade will induce local mode shape irregularities. These are then captured in the CWT by significantly magnified transform coefficients, thus providing level 3 damage identification. However, studies in [7], for example, suggest that the effectiveness of CWT for damage identification is typically increased when the derivatives of the mode 
shapes are examined. This can easily be exploited since

$$
\int_{\mathbb{R}} x^{k} \psi(x) \mathrm{d} x=0, \quad k=0,1, \ldots, n-1,
$$

in which it is stated how a wavelet has $n$ vanishing moments when $k=n-1$. This means that a wavelet with $n$ vanishing moments is orthogonal to polynomials up to degree $n-1$, thus resulting in zero-valued wavelet coefficients for these polynomials. By rewriting Equation (1) into a convolution, the following is obtained:

$$
W f(a, b)=\left(\frac{\mathrm{d}^{n}}{\mathrm{~d} x^{n}} f * \psi_{a}^{\prime}\right)(b)=\left(f * \frac{\mathrm{d}^{n}}{\mathrm{~d} x^{n}} \psi_{a}^{\prime}\right)(b) \because \psi_{a}^{\prime}(x)=\frac{1}{\sqrt{a}} \psi^{*}\left(\frac{-x}{a}\right),
$$

hence the CWT of the $n$th derivative of the mode shape is found directly as a convolution of the original mode shape and a wavelet with $n$ vanishing moments.

In the present study, several wavelet types were tested, and it was found that a real-valued Gaussian wavelet with four vanishing moments, i.e.

$$
\psi(x)=\frac{\mathrm{d}^{4}}{\mathrm{~d} x^{4}}\left(C_{4} e^{-x^{2}}\right)=4 C_{4} e^{-x^{2}}\left(4 x^{4}-12 x^{2}+3\right),
$$

yields the best results in the damage identification analyses of the SSP $34 \mathrm{~m}$ blade. Consequently, this particular wavelet was used. The constant in Equation (5) is found such the Euclidean norm $\left\|\mathrm{d}^{4} \theta(x) / \mathrm{d} x^{4}\right\|^{2}=1$. By employing scaling and position variables, the wavelet is dilated and translated accordingly.

\subsubsection{Mode shape interpolation}

When conducting modal analysis to derive spatial mode shapes, a finite, and often reduced, number of measurement points are employed, resulting in introduction of discontinuities in the CWT due to low resolution. In order to overcome this issue, a smoothing scheme was applied in the form of cubic spline interpolation, where the original mode shape signals were oversampled with an increment of $10 \mathrm{~mm}$.

\subsubsection{Mode shape extrapolation}

Since the CWT, cf. Equation (1), is an infinite integral transformation, adverse boundary effects will be introduced when employing the finite mode shape signal and wavelet function. These boundary effects will yield significant increases/decreases in the CWT coefficients at the boundaries which may conceal actual damage-induced CWT coefficient extrema. Therefore, an extrapolation scheme was included for removal of these boundary effects by shifting them to outside the interval in which the mode shapes are defined. Several studies, see e.g. [11], suggest using cubic spline extrapolation, but in the present study, it was found that linear extrapolation utilizing the two outermost points at each end of the smoothed signal yields the most effective removal of adverse boundary effects. It is important to notice that this approach of course depends on the spatial resolution in the smoothed signals.

\subsection{CWT of SSP $34 \mathrm{~m}$ mode shapes}

By convolving the SSP 34 m mode shapes with the chosen Gaussian wavelet, CWTs were derived for both damaged and undamaged states. By this means, it was found that valid damage identification can only be obtained when inspecting the CWTs of the flapwise components of mode shape 8, which 
is a combination of the 4th flapwise and 3rd edgewise bending modes, see Table 1 and Figure 4 . In Figure 5, this is depicted based on the full series measurements, that is, state 1 and 5. It was observed that similar results were obtained consistently for the other measurement states.

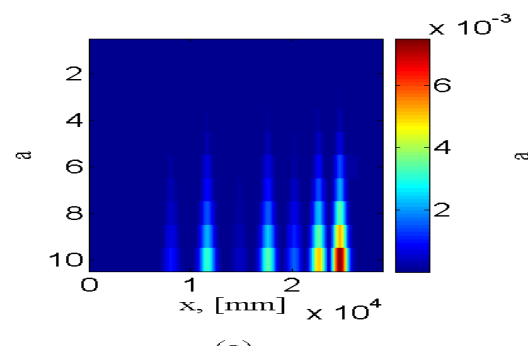

(a)

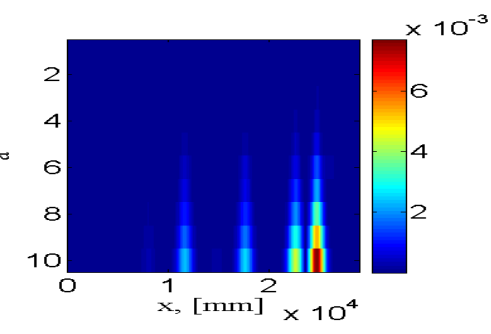

(b)

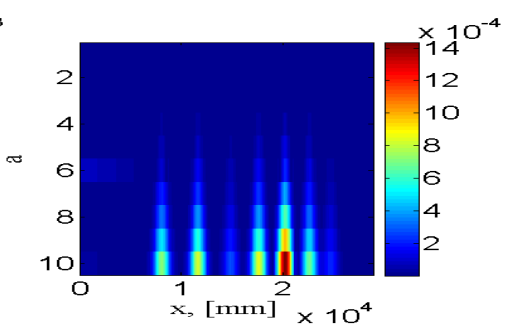

(c)

Figure 5 : CWTs of the 8th mode flapwise components, with damage location $x \in[18,800 \mathrm{~mm} ; 20,000 \mathrm{~mm}]$. (a) Damaged (1st state). (b) Undamaged (5th state). (c) Difference between damaged and undamaged.

Evidently, the damage-induced irregularities are not sufficient for the post-damage CWT to work as an independent damage indicator. However, when the CWTs from the undamaged states are subtracted from the post-damage counterparts, the maximum CWT coefficients are obtained at $x \approx 20,000 \mathrm{~mm}$, where the damage is located. Thus, the debonding can be validly detected and localized. Regarding size assessment, it was observed that multiple distinct CWT peaks occur at nondamaged areas, making it troublesome to point out the peaks induced by the actual damage. Several studies, see e.g. [11], have suggested employing a statistical threshold value below which all CWT coefficients are set to zero for eliminating the non-damage CWT peaks. A similar statistical approach was tested in the present study, but it was found that a very fine measurement density is needed to provide a sufficient threshold value and therefore the approach was discarded.

As an alternative to visually inspecting the CWT plots for damage identification, a simple damage indicator was introduced for level 1 damage identification, i.e. detection. The indicator is inspired by the MAC typically used in modal analysis (Cross-MAC), leading to the damage index

$$
\mathrm{DI}_{a, i j}=\frac{\left|\beta_{a, i}^{T} \gamma_{a, j}\right|^{2}}{\beta_{a, i}^{T} \beta_{a, i} \gamma_{a, j}^{T} \gamma_{a, j}} \in[0 ; 1],
$$

where $\beta_{a, i}$ is the CWT coefficient vector of the undamaged reference mode shape $i$ at scale $a$, while $\gamma_{a, j}$ is the CWT coefficient vector of either pre- or post-damage mode shape $j$ at scale $a$, i.e. $\gamma \supseteq \beta$. By choosing state 5 as the undamaged reference and subsequently analyzing the flapwise components of mode shape $i=j=8$ at scales $a \in[5 ; 10]$, the DI values presented in the box-and-whisker plot in

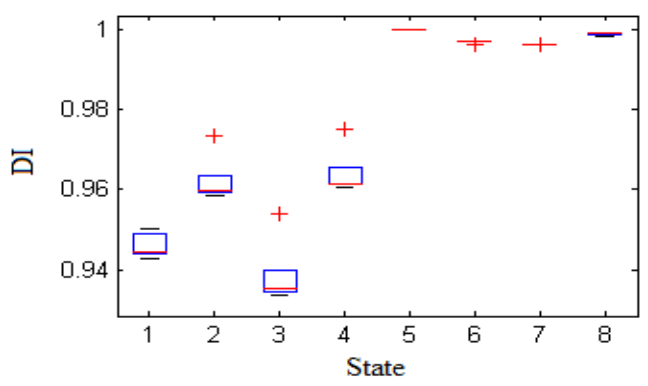

Figure 6: Box-and-whisker plot of DI values for flapwise components of mode shape 8, with state 5 as reference. The central mark in each box is the median, the edges of the box are the 25 th and 75 th percentiles, the whiskers extend to the most extreme data points not considered outliers ( \pm 2.7 times the spreading), and outliers are plotted individually. 
Figure 6 were derived. It is noticed that the results for $a \in[1 ; 4]$ were discarded in the analyses, as the high-frequency nature of these low scales increase the sensitivity towards measurement noise.

Evidently, the DI values decrease in the post-damage states, i.e. 1-4. The significance of these reductions are quantified through the box-and-whisker plot. Here, it is clearly seen that the DI value changes in the post-damage states are significant and cannot be categorized as measurement uncertainties, therefore implying that the changes must arise from the introduction of debonding.

\section{CONCLUSION}

The paper deals with modal and wavelet analysis-based damage identification in wind turbine blades. The studied damage identification method is based on modal analysis for derivation of structural mode shapes, which are subsequently signal-processed in the spatial domain and analyzed by means of onedimensional CWT. The method allows combined detection, localization, and assessment of structural damage. In the presented study, the abilities of the method were demonstrated by detection and localization of a $1.2 \mathrm{~m}$ trailing edge debonding of a $34 \mathrm{~m}$ blade. It was found that this type of damage could be validly detected and localized when comparing pre- and post-damage CWTs of the 8th blade mode. Regarding damage assessment in this mode, it was found that multiple distinct CWT peaks occur at non-damaged areas, thus it is troublesome to point out the peaks induced by the actual damage.

\section{ACKNOWLEDGMENTS}

The work was partly supported by EUDP (Danish Energy Technology Development and Demonstration Programme), grant number 64011-0084 "Predictive Structure Health monitoring of Wind Turbines".

The authors would like to thank DTU Wind Energy for giving access to the test object and research engineer Per Hørlyk Nielsen for his great assistance in setting up and conducting the experiment.

\section{REFERENCES}

[1] M. M. Khan, M. T. Iqbal, and F. Khan. Reliability and condition monitoring of a wind turbine. Proceedings of 18th Annual Canadian Conference on Electrical and Computer Engineering, 2005.

[2] S. Ataya and M. M. Z. Ahmed. Forms of discontinuities in $100 \mathrm{KW}$ and $300 \mathrm{KW}$ Wind Turbine Blades. Proceedings of 10th World Wind Energy Conference \& Renewable Energy Exhibition, 2011.

[3] F. M. Larsen and T. Sorensen. New Lightning Qualification Test Procedure for Large Wind Turbine Blades. Proceedings of International Conference on Lightning and Static Electricity, 2003.

[4] A. Rytter. Vibration Based Inspection of Civil Engineering Structures. Ph.D. Thesis, Aalborg University, Denmark, 1993.

[5] J. F. Skov, M. D. Ulriksen, K. A. Dickow, P. H. Kirkegaard, and L. Damkilde. On Structural Health Monitoring of Wind Turbine Blades. Proceedings of 10th International Conference on Damage Assessment of Structures, 2013.

[6] O. S. Salawu. Detection of structural damage through changes in frequency: a review. Journal of Engineering Structures, 19:718-723, September 1997.

[7] C. C. Ciang, J.-R. Lee, and H.-J. Bang. Structural health monitoring for a wind turbine system: a review of damage detection methods. Measurement Science and Technology, 19:122001, December 2008.

[8] M. D. Ulriksen, J. F. Skov, K. A. Dickow, P. H. Kirkegaard, and L. Damkilde. Damage Identification in Wind Turbine Blades - A Modal and Wavelet Analysis-based Method. To appear.

[9] L. Doliński and M. Krawczuk. Damage detection in turbine wind blades by vibration based methods. Journal of Physics: Conference Series, 181:012086, September 2009.

[10] SSP Technology. SSP 34m. http://www.ssptech.com/solutions/ssp-blade/ssp-34m.aspx. Accessed 14.03.2014.

[11] S. Loutridis, E. Douka, L. J. Hadjileontiadis, and A. Trochidis. A two-dimensional wavelet transform for detection of cracks in plates. Engineering Structures, 27:1327-1338, August 2005. 